Computer Science \& Engineering: An International Journal (CSEIJ), Vol.2, No.1, February 2012

\title{
ANALYSIS OF CONTENTION BASED METHOD FOR MAC LAYER IN WIRELESS NETWORKS
}

\author{
V.R.Azhaguramyaa ${ }^{1}$, S.J.K. Jagadeesh Kumar $^{2}$ \\ ${ }^{1}$ PG Student, ${ }^{2}$ Professor \& Head \\ ${ }^{1,2}$ Department of Computer Science and Engineering, Sri Krishna College of Technology, \\ Coimbatore, India \\ ${ }^{1}$ vrazhaguramyaa@gmail.com, 2 jagadeesh_sk@rediffmail.com
}

\begin{abstract}
Quality of Service $(Q o S)$ is the ability to guarantee a certain level of performance to a data flow ie., guaranteeing required bit rate, delay, etc. IEEE $802.11 \mathrm{a} / \mathrm{b} / \mathrm{g}$ networks do not provide QoS differentiation among multimedia traffic. QoS provisioning is one of the essential features in IEEE 802.11e. It uses Enhanced Distributed Channel Access (EDCA) which is a contention-based channel access mode to provide QoS differentiation. EDCA works with four Access Categories (AC). Differentiation of Access Categories are achieved by differentiating the Arbitration Inter-Frame Space (AIFS), the initial contention window size (CWmin), the maximum contention window size (CWmax) and the transmission opportunity (TXOP). However AIFS, CWmin, CWmax are considered to be fixed for a given AC, while TXOP may be varied. A TXOP is a time period when a station has the right to initiate transmissions onto the wireless medium. By varying the TXOP value among the ACs the QoS optimization- throughput stability and minimum delay is achieved. EDCA has many advantages such as it fully utilizes the channel bandwidth, and does not require centralized admission control and scheduling algorithms over the contention-free access mode.
\end{abstract}

\section{KEYWORDS}

EDCA, IEEE 802.11e, Medium Access Control, QoS optimization, Quality of Service, TXOP limit.

\section{INTRODUCTION}

\subsection{IEEE 802.11}

In recent years, among the various WLAN technologies available in the market, Due to the low cost, ease of deployment, and mobility support, IEEE 802.11 standard wireless LANs has gradually become the preferred technology for wireless Internet and Intranet access. IEEE 802.11 network can be easily deployed in hot-spot zones of airports, hotels, stock markets, residence homes and other places. It was basically developed to serve best effort data services, so there are many inherent QoS limitations in the base standard.The base version of the standard has subsequent amendments. With the increasing demand for real-time multimedia application support, a new standard amendment, IEEE 802.11e, has been specified [7]. It aims to support QoS by providing differentiated classes of service in the medium access control (MAC) layer so that it can deliver time-critical multimedia traffic in addition to traditional data packets. 


\subsection{IEEE 802.11e}

IEEE 802.11e-2005 or 802.11e [7] is an approved amendment to the IEEE 802.11 standard that defines a set of Quality of Service enhancements for wireless LAN applications through modifications to the Media Access Control (MAC) layer. The standard is considered of critical importance for delay-sensitive applications, such as Voice over Wireless LAN and streaming multimedia.

\subsection{IEEE 802.11e MAC operation}

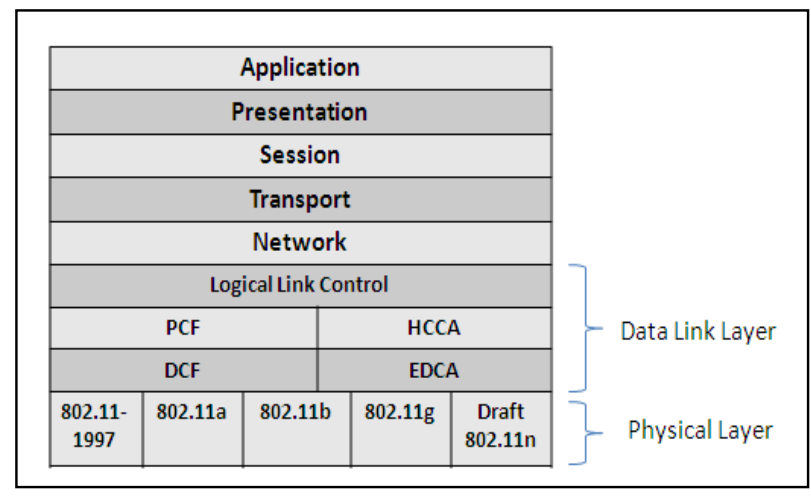

Figure 1. OSI model of IEEE 802.11e

The 802.11e has new coordination function which enhances the DCF and the PCF: the hybrid coordination function (HCF). Within the HCF, there are two methods of channel access, similar to those defined in the legacy 802.11 MAC: HCF Controlled Channel Access (HCCA) and Enhanced Distributed Channel Access (EDCA) which is illustrated in Figure 1.Both EDCA and HCCA define Access Categories. To protect high priority data from low priority data is the primary purpose of QoS. In some scenarios data needs to be protected from other data of the same class. Admission Control in EDCA address these types of problems. The available bandwidth is published by the AP in beacons. Before adding more traffic, clients can check the available bandwidth.

\subsection{Importance of access categories in 802.11e}

The 802.11e MAC supports the access categories which are listed in Table 1.

Table 1. Access categories

\begin{tabular}{|l|l|}
\hline \multicolumn{1}{|c|}{ Access categories } & \multicolumn{1}{c|}{ Description } \\
\hline AC_VO(Voice) & Voice traffic and network control belong to AC_VO. \\
\hline AC_VI(Video) & Video and video/controlled load belong to AC_VI. \\
\hline AC_BE(Best Effort) & Best effort (E-mail) and video/excellent effort belong to AC_BE. \\
\hline AC_BK(Background) & Background (Uninvited) traffic will come under AC_BK.. \\
\hline
\end{tabular}


Computer Science \& Engineering: An International Journal (CSEIJ), Vol.2, No.1, February 2012

\subsection{Other 802.11e specifications}

In addition to HCCA, EDCA and TXOP, 802.11e specifies additional optional protocols for enhanced 802.11 MAC layer QoS:

\subsubsection{Automatic power save delivery}

While comparing with 802.11 Power Save Polling, Automatic power save delivery is a more efficient power management method. Information about 802.11 Power Save Mode overview is available in [13], an analysis of unscheduled and scheduled automatic power save delivery (APSD) is available in [14] and a comparison of both available in [15]. Mostly all new 802.11 stations already support a power management mechanism similar to APSD, which is very useful for a VoIP phone due to data rates are roughly the same in both directions. At first voice data is sent to the access point, the AP is triggered to send the buffered voice data in the other direction. Next voice data has to be sent to the access point when the VoIP phone enters a doze state.

\subsubsection{Block acknowledgments}

An entire TXOP to be acknowledged in a single frame can be allowed using Block Acknowledgements. This is useful when longer TXOPs are specified and provides less protocol overhead.

\subsubsection{NoAck}

There are two values in service class for frames to be sent during QoS mode: QosAck and QosNoAck. Frames with QosNoAck are not acknowledged. Using this, retransmission of highly time-critical data is avoided.

\subsubsection{Direct Link Setup}

Within a basic service set, direct station-to-station frame transfer can be allowed using direct link setup which is designed for consumer use, where station-to-station transfer is more commonly used.

To accomplish the same goal Microsoft's Virtual Wi-Fi initiative is designed. Using Virtual WiFi gamers can be connected to wireless while accessing the Internet through an AP by allowing station adapters to have multiple MAC addresses.

\subsection{Importance of TXOP LIMIT}

There have been various Frequency-based approaches are available for QoS optimization but they incur high computational complexity because modeling the AIFS, CWmin and CWmax values require solving non-linear equation systems that are extremely computationally demanding and not suitable for real-time applications. QoS optimization in contention-free mode requires centralized admission and scheduling algorithms, thus not flexible. In addition, it may not fully utilize the channel bandwidth, because it relies on resource reservations, which are typically made for worst case scenarios. The Enhanced Distributed Channel Access mode is able to provide QoS optimization by easily controlling the TXOP. 


\subsection{QoS optimization}

The process of QoS optimization described in Figure 2. Initially heterogeneous traffic reaches the MAC layer including voice, video, best effort, background and they are mapped to the corresponding Access Categories. Then all frequency-related parameters of various Access Categories including AIFS, CWmin, and CWmax are fixed by controlling the Transmission Opportunity Limit parameter the higher priority traffic has a higher chance of being sent and waits a little less before it sends its packet, on average, than a station with low priority traffic.

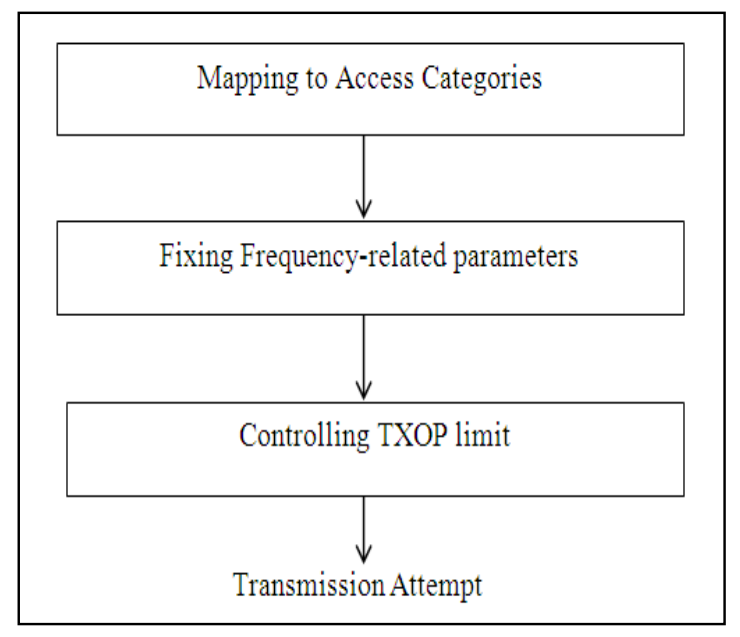

Figure 2. QoS optimization process

\section{RELATED WORK}

H. S. Chhaya et al. present an analytical model of the DCF from the point of view of collision avoidance (CA) with a backoff procedure and capture effect in [6].

The backoff time was assumed to follow a geometric distribution with a parameter related to the average value of all the backoff times. Theoretical and simulation results were obtained and some improvements were also proposed by F. Cali et al in [3]-[5].

Model proposed by Nada Chendeb Taher et al. considers the $802.11 \mathrm{e}$ contention free burst (CFB) mode which allows a given station to transmit a burst of frames without contention during a given transmission opportunity limit (TXOPLimit) time interval. The effect of TXOPLimit differentiation parameter on the global performance is studied thoroughly in the paper [10]. They have developed a new and complete analytical model using a four-dimensional discrete time Markov chain in general network conditions.

The number of transmissions per packet was assumed to be geometrically distributed, by Y. C. Tay et al. with an average backoff time equal to half of the contention window (CW) size [16]. All of these works concentrated on the analysis of legacy DCF. However they do not concentrate on EDCA mechanism.

Bellalta. B.et al investigated the basic values of EDCA parameters which should be changed to perform the QoS optimization [1]. Their work is mainly based on the frequency of acquiring transmission opportunities parameters (ie., CWmin, CWmax, AIFS) and they have concentrated, 
Computer Science \& Engineering: An International Journal (CSEIJ), Vol.2, No.1, February 2012

to maximize the elastic(BE) throughput while assuring the bandwidth-delay requirements of the rigid flows $(\mathrm{VO})$.

Zhen-ning, Kong et al. analyzed the performance of contention-based channel access in IEEE 802.11e [17] and they have produced the markov chain model of one Access Category per station. They have concentrated on AIFS in the Enhanced Distributed Channel Access and produced the formula for calculating AIFS.

The above works [1], [17] focus only on the frequency of acquiring transmission opportunities parameters and not the duration of transmission opportunity parameter and not considering the throughput of video traffic (VI).

In this paper we propose a new optimization algorithm which is the modification of EDCA and that new algorithm provides per stream QoS which is not available in EDCA [2] and it is achieved by tuning the duration of transmission opportunity parameter called TXOP limit. This new work follows the implementation details outlined by, Khaled A. Shuaib.

The author specified the cell structure of wireless networks, important parameters needed for creating the simulation and the packet interval of various access categories used in Qualnet simulation tool [9]. The author also stated various simulation scenarios with variable number of stations and with heterogeneous applications and analyzed their results.

\section{Proposed SCHEME}

\subsection{EDCA}

The Enhanced Distributed Channel Access (EDCA) mechanism of 802.11e extends the basic 802.11 DCF algorithm with Quality of Service capabilities. In EDCA mode, packets are categorized into prioritized classes, called access categories (ACs). Traffic sessions compete with each other for the wireless medium. In EDCA mode, airtime allocation among traffic sessions in different ACs is differentiated by assigning each AC with different EDCA parameters. Differential allocation of airtime to different ACs is essential for QoS-enabled applications. For example, some traffic sessions belonging to the video AC may need to be allocated larger fractions of the airtime than traffic sessions belonging to the best-effort AC.

\subsection{EDCA parameters}

There are two sets of EDCA parameters that can achieve airtime differentiation. The first set controls the frequency of acquiring a transmission opportunity on the wireless medium. The second set controls the duration of an acquired transmission opportunity.

\subsubsection{Frequency of transmission opportunities}

The frequency of transmission opportunities is determined through three parameters:

- $\quad$ Arbitration Inter Frame Space (AIFS)

- Minimum Contention Window Size (CWmin)

- Maximum Contention Window Size (CWmax)

Each AC maintains a contention window size variable $(\mathrm{CW})$, which is initialized to $\mathrm{CWmin}$. The $\mathrm{CW}$ is incremented after transmission failures until it reaches CWmax, and is reset to CWmin 
Computer Science \& Engineering: An International Journal (CSEIJ), Vol.2, No.1, February 2012

after a successful transmission. To avoid collisions, a backoff timer is independently chosen from the range $[0, \mathrm{CW}]$ for each $\mathrm{AC}$. Since smaller $\mathrm{CWmin}$ and $\mathrm{CWmax}$ generally lead to smaller $\mathrm{CW}$ values, they result in shorter backoff timer and higher transmission opportunity frequency. Moreover, the backoff timer is decremented once the wireless medium is sensed idle for at least AIFS seconds. Smaller AIFS values enable wireless stations to start decrementing backoff timers earlier, and thus increase the transmission opportunity frequency.

\subsubsection{Duration of transmission opportunity}

The maximum allowed duration for each acquired transmission opportunity is determined by a parameter called,

\section{- $\quad$ TXOP limit.}

Once a station acquires a transmission opportunity, it may transmit multiple frames within the assigned TXOP limit. Assigning different TXOP values to ACs, therefore, achieves differential airtime allocations [2]. Controlling the TXOP limit allows us to derive a simple, closed-form equation for the effective airtime.

\subsection{EDCA mechanism}

The contention-based channel access of HCF is also referred to as EDCA. A new concept, transmission opportunity (TXOP), is introduced in IEEE 802.11e. A TXOP is a time period when a station has the right to initiate transmissions onto the wireless medium. TXOP is defined by a starting time and a maximum duration.

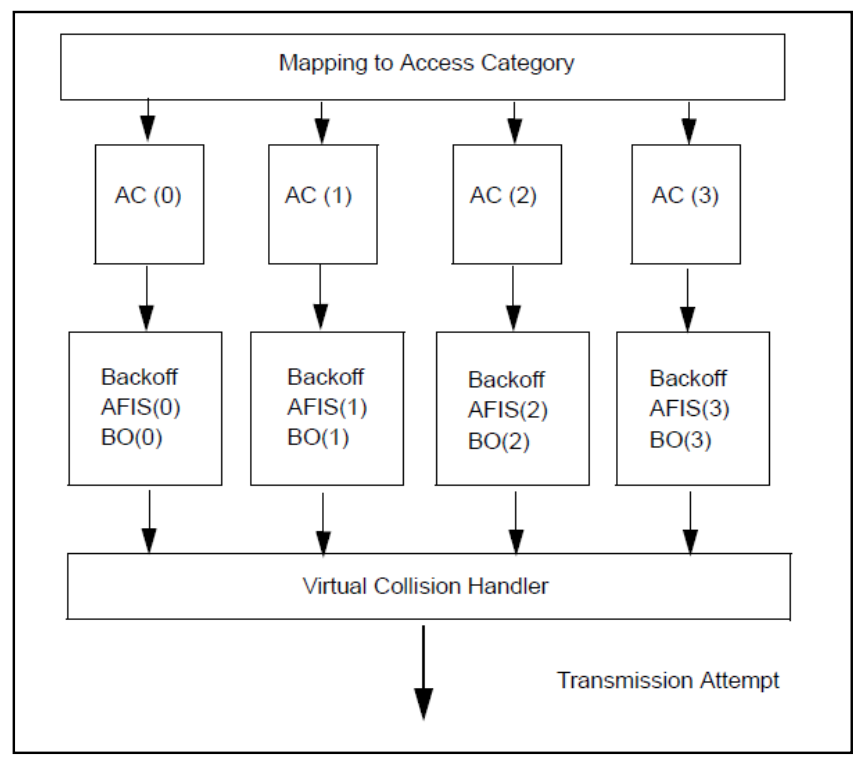

Figure 3. EDCA 


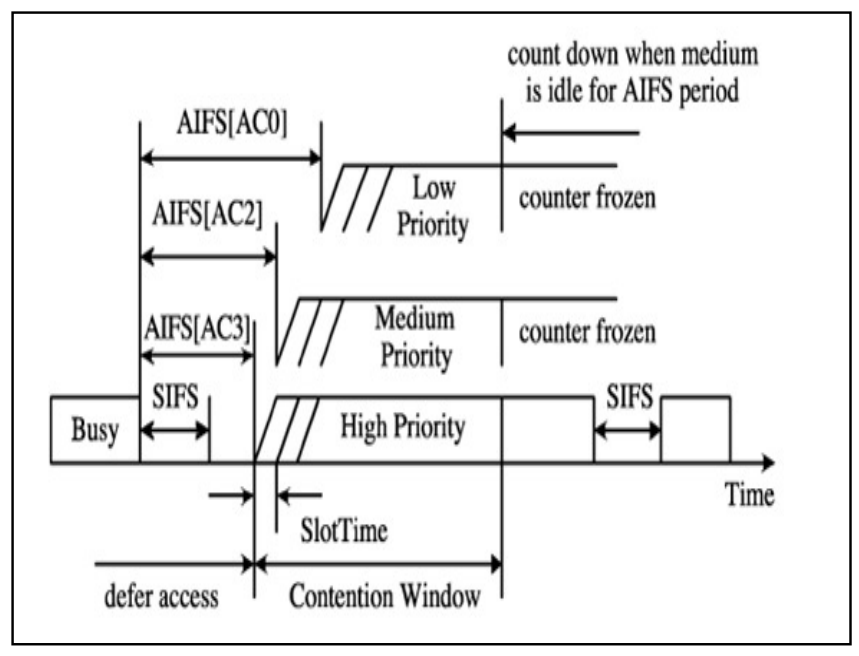

Figure 4. IEEE 802.11e EDCA parameters

A station cannot transmit a frame that extends beyond a TXOP. If a frame is too large to be transmitted in a TXOP, it must be fragmented into smaller frames. EDCA works with four Access Categories (ACs), which are virtual DCFs [11] as shown in Figure 3, where each AC achieves a differentiated channel access. This differentiation is achieved through varying the amount of time; a station would sense the channel to be idle, and the length of the contention window for a backoff. Differentiated ACs is achieved by differentiating AIFS [17] as shown in Figure 4, the initial window size and the maximum window size. That is, for AC $\mathrm{i}$ (where $\mathrm{i}$ is 0,1 , 2 , or 3), the initial backoff window size is CWmin[i], the maximum backoff window size is $\mathrm{CWmax}[\mathrm{i}]$, and the arbitration inter-frame space is AIFS[i]. For $0 \leq \mathrm{i} \leq \mathrm{j} \leq 3$, we have CWmin[i] $\geq \mathrm{CWmin}[\mathrm{j}]$ and CWmax[i] $\geq \mathrm{CWmax}[\mathrm{j}]$, and AIFS[i] $\geq$ AIFS[j] and at least one of the above inequalities must be "not equal to"[2]. In other words, the EDCA employs AIFS[i], CWmin[i], and CWmax[i] (all for $\mathrm{i}=0, \ldots, 3$ ) instead of DIFS, minCW and maxCW, respectively [8]. If one $\mathrm{AC}$ has a smaller AIFS or min $\mathrm{CW}$, the traffic of AC has a better chance to access the wireless medium earlier, thus providing the QoS effect.

\subsection{QOS optimization in IEEE 802.11e using EDCA}

Table 2. Access categories and precedence levels

\begin{tabular}{|l|l|}
\hline \multicolumn{1}{|c|}{ Mac layer services } & \multicolumn{1}{c|}{ Precedence } \\
\hline AC[0]-BACKGROUND & 0 \\
\hline AC[1]-BEST EFFORT & 1,2 \\
\hline AC[2]-VIDEO & $3,4,5$ \\
\hline AC[3]-VOICE & 6,7 \\
\hline
\end{tabular}


Table 3. Values of EDCA parameters

\begin{tabular}{|c|c|c|c|c|}
\hline AC & AIFSN & $\begin{array}{c}\text { TXOP } \\
(\mathbf{m s})\end{array}$ & CWmin & CWmax \\
\hline $\mathrm{AC}[0]-\mathrm{BK}$ & 7 & 0 & $C W \min$ & $C W \max$ \\
\hline $\mathrm{AC}[1]-\mathrm{BE}$ & 3 & 0 & $C W \min$ & $C W \max$ \\
\hline $\mathrm{AC}[2]-\mathrm{VI}$ & 2 & 6.016 & $C W \min / 2$ & $C W \min$ \\
\hline $\mathrm{AC}[3]-\mathrm{VO}$ & 2 & 3.264 & $C W m i n / 4$ & $C W m i n / 2$ \\
\hline
\end{tabular}

Access categories of IEEE 802.11e mapped to various precedence levels in the EDCA implementation which is available in Table 2. Each frame from the higher layer arrives at the MAC along with a specific priority (precedence) value. Four access categories ACs are available with 802.11e [12]. Frame with Priority value is mapped into an $\mathrm{AC}$ when it is arriving at the MAC. The Table 3 specifies the values of EDCA parameters which belong to different access categories of IEEE 802.11e. To achieve differentiation, instead of using fixed DIFS as in the DCF, an AIFS is applied, where the AIFS for a given AC is determined by the following equation:

$$
\text { AIFS }=\text { SIFS }+ \text { AIFSN } * \text { aSlotTime }
$$

Where AIFSN is AIFS Number and determined by the AC and physical settings, and aSlotTime is the duration of a time slot [1]. The highest priority will be given to the AC with the smallest AIFS. The CWmin value is 31 and the CWmax value is 1023 .

\subsection{TXOP LIMIT- The controlling knob}

Consider $\mathrm{S}$ wireless stations compete for the shared air medium of a wireless LAN using the IEEE 802.11e EDCA protocol. These wireless stations transmit data to/from the base station at different bit rates, and the rate differentiation is achieved by varying the TXOP limits for individual wireless stations [2]. In optimization problem, it is a need to determine the total effective airtime (EA) of the wireless medium so that it can be divided among stations, and to avoid over/under allocation of the wireless medium. The virtual transmission time $\mathrm{v}_{\mathrm{j}}$ as the time duration between the $\mathrm{j}$-th and the $(\mathrm{j}+1)$-th successful transmissions is defined.

Each virtual transmission consists of three periods:

- Idle

- Collision

- Transmission

The idle period happens when all wireless stations are waiting for their backoff timers to expire. The collision period happens when more than one station initiate transmissions. The idle and collision periods may appear more than once in a virtual transmission, while a single transmission period occurs during a virtual transmission.

Let consider $E[x]$ to denote the average transmission opportunity limit for all wireless stations, and $E[v]$ to denote the average virtual transmission time. Then, the effective airtime can be given by:

$$
E A=E[x] / E[v]
$$

That is, the effective airtime is given by the ratio of the actual (useful) transmission time to the total transmission time after including all contention overheads, which are modeled by the virtual 
Computer Science \& Engineering: An International Journal (CSEIJ), Vol.2, No.1, February 2012

transmission time. Next $E[v]$ will be computed, by averaging the durations of collision and idle periods during a virtual transmission time.

Let denote the number of collisions in a virtual transmission time by $C$, define $i_{k}$ to be the duration of the $k$-th idle period, and similarly, $c_{k}$ to be the duration of the $k$-th collision period. Then $E[v]$ is given by:

$$
E[v]=E[C]\left(E[c]+t_{d}+t_{s}+t_{a}\right)+(E[C]+1) E[i]+E[x]+t_{d}
$$

Where,

$t_{d}$ is the distributed inter-frame space (DIFS),

$t_{s}$ is the short inter-frame space (SIFS),

$t_{a}$ is the average time of sending an acknowledgment.

From the equation (3) it is found that optimal solution for airtime differentiation comes from controlling the TXOP limit and by fixing the frequency of transmissions opportunities parameters.

\section{EXPERIMENTAL RESULTS}

Simulation Scenario consists of 1 Access Point and 24 wireless stations. Scenario has the following properties [9]:

Simulation tool -QualNet 5.0.2

PHY Layer Model - IEEE 802.11b

Bandwidth - 20MHZ (min)

Antenna Height (PHY) - 1.5 meters

Terrain Space $-500 * 500$ meters

Simulation time -100 seconds

Items to send -0 (denotes packets will be send upto end of the simulation)

Scheduling algorithm used to select service classes - Strict Priority

Scheduling algorithm used inside the service class- FIFO

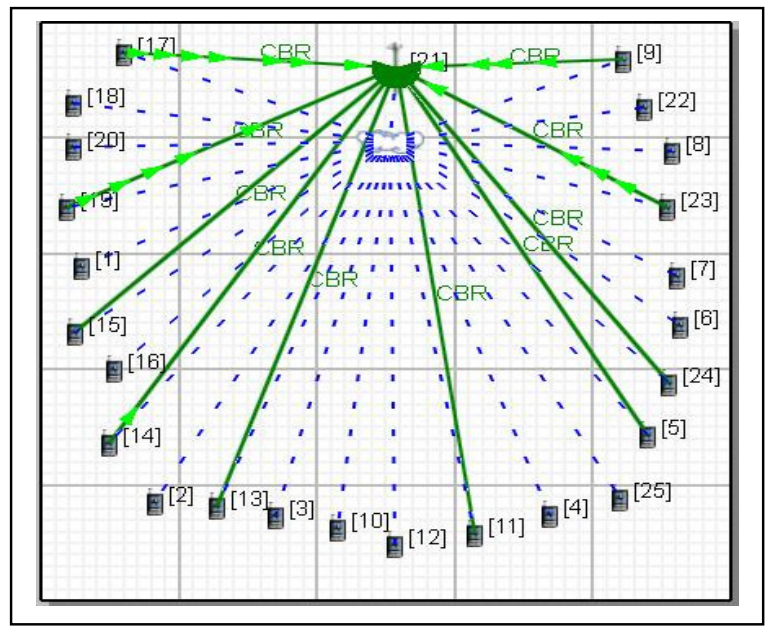

Figure 5. Scenario Simulation 
Computer Science \& Engineering: An International Journal (CSEIJ), Vol.2, No.1, February 2012

Table 4. Traffic precedence levels

\begin{tabular}{|c|c|c|}
\hline Source & Destination & Precedence level \\
\hline 19 & 21 & 7 \\
\hline 23 & 21 & 7 \\
\hline 15 & 21 & 0 \\
\hline 24 & 21 & 0 \\
\hline 13 & 21 & 1 \\
\hline 11 & 21 & 1 \\
\hline 17 & 21 & 7 \\
\hline 9 & 21 & 7 \\
\hline 14 & 21 & 3 \\
\hline 5 & 21 & 3 \\
\hline
\end{tabular}

In 24 wireless stations 10 stations are generating traffic and that traffic are assigned to various traffic categories of IEEE 802.11e. All are using CBR application and they are varied by assigning the precedence levels of EDCA. Table IV specifies the traffic generating nodes and their precedence levels.

The Figure 5 shows the simulation of created scenario and its progress. During simulation, traffic is generated from the listed nodes and reaches the Access Point which is node no. 21. It is the Uplink Scenario.

\subsection{Throughput analysis}

Throughput is calculated for both Access point and wireless stations using the following formula:

\section{Access Point}

Throughput $=(($ Total no. of bytes received $* 8) /$ Session Duration $)$

\section{Wireless Stations}

Throughput $=(($ Total no. of bytes sent $* 8) /$ Session Duration $)$

\subsubsection{Access Point Throughput}

Figure 6 shows the throughput of server, the Access point node (21) is the server, throughput of 21 is very high because it always receives packets from the high priority traffic because of the TXOP variation. This result is taken from the statistics (.stat) file of created scenario. 


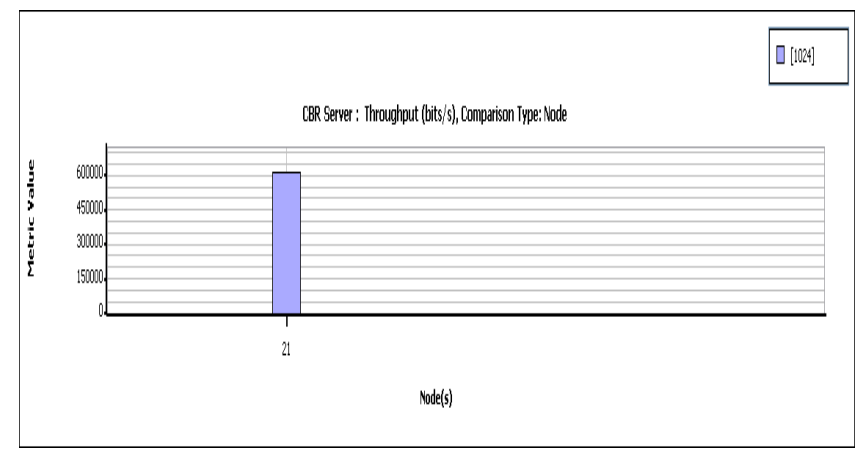

Figure 6. Access point throughput analysis

\subsubsection{Wireless stations Throughput}

Fig. 7 shows the throughput of wireless stations which generated by various traffic. The analysis says that the throughput of nodes which generate high priority traffic $(9,17,19$ and 23$)$ is very high because of the TXOP variation.

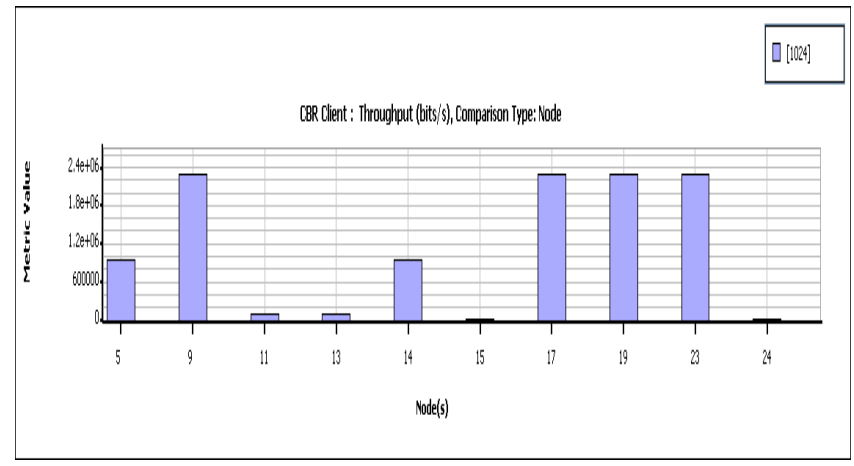

Figure 7. Wireless stations throughput analysis

\subsection{Average End-To-End delay analysis}

Average end-to-end delay is calculated at the Access Point using the following formula.

CBR Avg. End-to-end delay = (Sum of the delays of each CBR packet received $) /$ (No. of CBR Packets received)

Fig. 8 shows the average end-to-end delay of the Access Point. From the statistics (.stat) file of created scenario, it is clear that the delay of high priority traffic is comparatively less than the low priority traffic. 


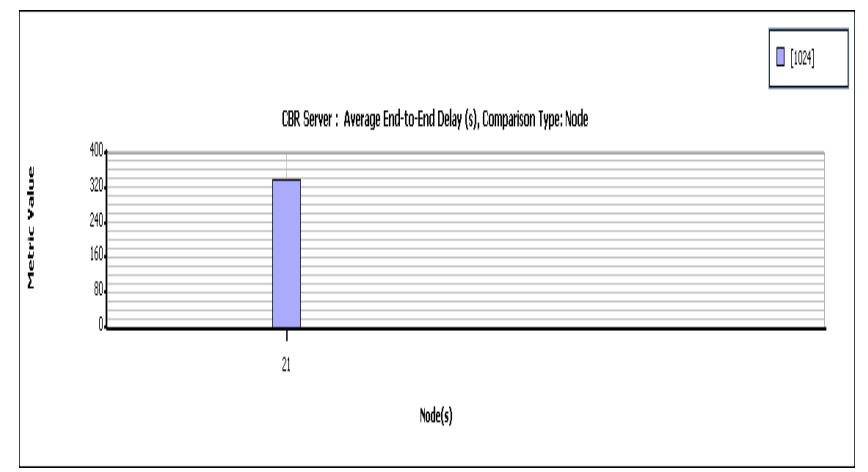

Figure 8. Average End-to-End delay analysis

\section{CONClusion}

The QoS optimization is provided by Enhanced Distributed Channel Access mode in IEEE 802.11e based Networks. With EDCA, packets are categorized into prioritized classes, higher priority traffic has a higher chance of being sent and waits a little less before it sends its packet, on average, than a station with low priority traffic. Using EDCA the quality improvement comes at negligible cost, because the optimal solution is computed using simple equations. EDCA is suited for networks which support link-layer traffic differentiation.

In future, the EDCA mechanism can be implemented for IEEE 802.16 based networks and the cross layering framework can also be included to improve the QoS optimization.

\section{REFERENCES}

[1] Bellalta. B., Cano. C., Oliver. M. and Meo. M., "Modeling the IEEE 802.11e EDCA for MAC Parameter Optimization", Het-Nets 06, Bradford, UK, September 2006.

[2] Cheng-Hsin Hsu, Mohamed Hefeeda, "A Framework for Cross-Layer Optimization of Video Streaming in Wireless Networks", ACM Transactions on Multimedia Computing, Communications and Applications, Vol. 7, No. 1, Article 5, January 2011.

[3] F. Cali, M. Conti, and E. Gregori, "IEEE 802.11 wireless LAN: Capacity analysis and protocol enhancement," in Proc. IEEE INFOCOM'98, vol.1, Mar. 1998, pp. 142-149.

[4] F. Cali, M. Conti, and E.Gregori, "IEEE 802.11 protocol: Design and performance evaluation of an adaptive backoff mechanism,” IEEE J. Select. Areas. Commun., vol. 18, pp. 1774-1786, Sept. 2000.

[5] F. Cali, M. Conti and E. Gregori, "Dynamic tuning of the IEEE 802.11 protocol to achieve a theoretical throughput limit," IEEE/ACM Trans. Networking, vol. 8, pp.785-799, Dec. 2000.

[6] H. S. Chhaya and S. Gupta, "Performance modeling of asynchronous data transfer methods of IEEE 802.11 MAC protocol," Wireless Networks, vol. 3, pp. 217-234, 1997.

[7] IEEE Std 802.11e; Wireless LAN Medium Access Control (MAC) and Physical Layer (PHY) specifications; Amendment: Medium Access Control (MAC) QoS Enhancements, IEEE Std 802.11e2005.

[8] Jochen Schiller, "Mobile Communications", Second Edition, Pearson Education, 2003.

[9] Khaled A. Shuaib, "A Performance Evaluation Study of WiMAX Using Qualnet" Proceedings of the World Congress on Engineering 2009 Vol. I WCE 2009, July 1-3, 2009.

[10] Nada Chendeb Taher, Yacine Ghamri-Doudane, Bachar El Hassan and Nazim Agoulmine, "An accurate analytical model for 802.11e EDCA under different traffic conditions with contention-free bursting", Journal of computer Networks and Communications, March 2011. 
Computer Science \& Engineering: An International Journal (CSEIJ), Vol.2, No.1, February 2012

[11] Saurabh Sehrawat, Revoti Prasad Bora, Dheeraj Harihar, "Performance Analysis of QoS supported by Enhanced Distributed Channel Access (EDCA) mechanism in IEEE 802.11e," International Journal of Computer Science, April 2006

[12] "Windows 7 adds native Virtual WiFi technology from Microsoft Research". 16 May 2009. Retrieved 2010-07-07.

[13] X.Perez-Costa, D.Camps-Mur and T.Sashihara, "Analysis of the Integration of IEEE 802.11e Capabilities in Battery Limited Mobile Devices", IEEE Wireless Communications Magazine, special issue on Internetworking Wireless LAN and Cellular Networks, Volume 12, Issue 6, December 2005.

[14] X.Perez-Costa and D.Camps-Mur, "IEEE 802.11e QoS and power Saving feature: Overview and Analysis of Combined Performance", IEEE Wireless Commnications Magazine, Vol. 17, Issue 4, August 2010.

[15] X.perez-Costa, D.Camps-Mur and Albert Vidal, "On the Distributed Power Saving Mechanisms of Wireless LANs 802.11e U-APSD vs 802.11 Power Save Mode", Elsevier Computer Networks Journal (CN), Volume 51, Issue 9, June 2007.

[16] Y. C. Tay and K. C. Chua, “A capacity analysis for the IEEE 802.11 MAC protocol,"Wireless Networks, vol. 7, pp. 159-171, July 2001.

[17] Zhen-ning Kong, Danny H. K. Tsang, Brahim Bensaou and Dayun Gao, "Performance Analysis of IEEE 802.11e Contention-Based Channel Access", IEEE Journal On Selected Areas In communications, Vol. 22, No. 10, December 2004.

[18] http://www.qualnet.com

\section{Authors}

Ms.V.R.Azhaguramyaa has received her Bachelor of Engineering degree in Computer Science and Engineering under Anna University, Chennai in 2009. She is currently pursuing Master of Engineering degree in Computer Science and Engineering under Anna University, Coimbatore, India. Her areas of interests are Mobile Computing, Next Generation Wireless Networks.

Prof. S.J.K. Jagadeesh Kumar received his B. E degree from Madurai Kamaraj University, Tamilnadu, India in 1988 and M.E. degree from Bharathiyar University in 1998. Since 2006, he has been working as Professor in the department of Computer Science and Engineering at V.L.B. Janakiammal College of Engineering and Technology, Coimbatore, India. He was instrumental in receiving funding from various government agencies. His research interest includes WiMAX, Mobile Computing, and Wireless Sensor Networks. He is a member of ACM (USA), ISTE, CSE and ACS.
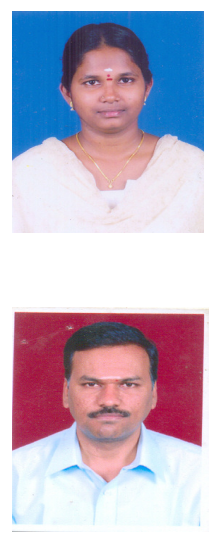